\title{
Experiments on high energy reactions in the diffractive regime at LHC
}

\author{
Stefan Tapprogge* \\ Helsinki Institute for Physics, \\ c/o CERN, Route de Meyrin, \\ CH-1211 Geneva 23, Switzerland \\ E-mail: Stefan.Tapprogge@cern.ch
}

\begin{abstract}
In a few years, the LHC will push the energy frontier in accelerator physics significantly further, with the primary goal of obtaining a better insight in the fundamental constituents of matter and their interactions, e.g. the understanding of the origin of the electroweak symmetry breaking. Due to its capability of colliding various beam species, it will also offer unique possibilities for further studies of the strong interaction, in as yet uncovered kinematical regions. In order to exploit the machine capabilities best, an extension of the coverage of the approved detectors in the forward region (small scattering angles wrt the beam) is highly desirable. This contribution discusses the physics motivation, the baseline machine and experiment layout and as well as possibilities for extensions of the detector coverage.
\end{abstract}

\section{Introduction}

The LHC is primarily designed to be a discovery machine, providing proton-proton collisions at the highest center-of-mass energy with a very large luminosity. It will however also be able to provide nucleus-nucleus and proton-nucleus collisions, again at the highest center-of-mass energies, offering a unique facility for the study of the strong interaction.

Amongst the primary goals for $p p$ collisions is the understanding of the origin of the electroweak symmetry breaking, which could manifest in the observation of one (or more) Higgs boson(s). The LHC will also vastly extend the potential for discovery of new physics beyond the Standard Model, extending the mass scale up to several $\mathrm{TeV}$ for direct observations. In addition, the experiments are designed with the goal of performing precision measurements within the Standard Model (and as well of new processes - if found).

\footnotetext{
${ }^{*}$ Speaker.
} 


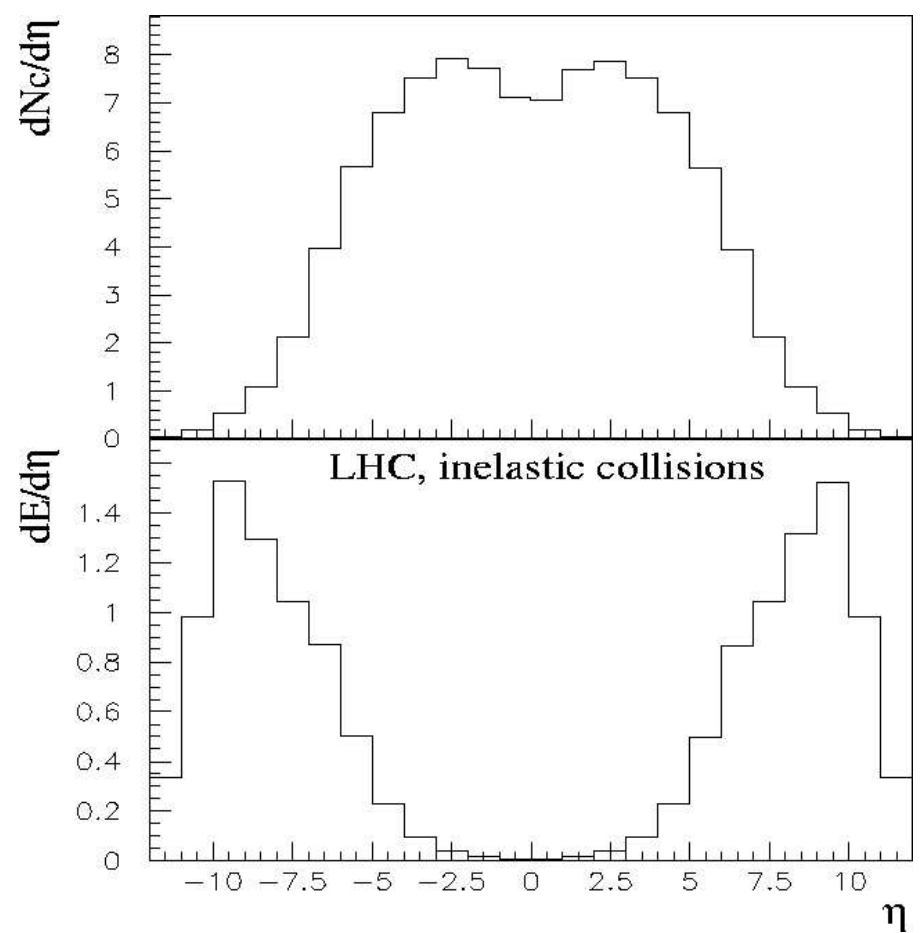

Figure 1: Particle production in $p p$ collisions at $\sqrt{s}=14 \mathrm{TeV}$ (from [1]).

The LHC will offer unique possibilities to study strong interaction properties at the (future) energy frontier in a variety of processes and thus probe further Quantum ChromoDynamics (QCD) as the fundamental theory of the strong interaction. These data are of importance (esp. for $p p$ collisions) to properly understand background processes for searches and precision measurements. Although a truely full acceptance detector is presently out of reach (as proposed in [1]), possibilities exist for a sizeable increase in physics coverage by adding additional components to one (or more) of the approved detectors at LHC. These additional components could provide a much better coverage of the forward region (the region of small scattering angles wrt the beam direction).

This contribution discusses the physics motivation for such extensions, followed by a brief description of the LHC machine and of the running scenarios presently foreseen. Next, an overview of the five approved LHC experiments and a summary of their baseline coverage is given. Finally, possibilities for extending the coverage in the forward region and related instrumentation aspects are discussed.

\section{Forward physics}

As shown in Fig. 1, $p p$ collisions show the highest multiplicities in the central region $(|\eta|<$ $5^{1}$ ). However the largest energies are found in the forward region (corresponding to very small scattering angles wrt the beam direction, e.g. $|\eta|>5$ implies $\theta<10 \mathrm{mrad}$ ). In order to observe particles in this region, detection has to occur at large distances from the

\footnotetext{
${ }^{1}$ Pseudo-rapidity $\eta=-\log \tan \frac{\theta}{2}$, coinciding for massless particles with the rapidity $y=\frac{1}{2} \log \frac{E+p_{z}}{E-p_{z}}$
} 
interaction point. As well be shown, it this region that is of most interest and at the same time the most challenging for experimental instrumentation. It is worth to note that a center-of-mass energy of $\sqrt{s}=14 \mathrm{TeV}$ for $p p$ collisions corresponds to an incident proton energy of about $10^{17} \mathrm{eV}$ in the laboratory frame.

The list of physics processes, which will benefit significantly from an enlarged acceptance at small angles wrt the beam, includes:

- total cross-section and elastic scattering,

- soft and hard diffractive scattering,

- properties of rapidity gaps,

- exclusive central production,

- event structure (energy flow, multiplicities, leading particle spectra, ...),

- low- $x$ phenomena,

- photon-nucleus interactions

and many more. In the following, more details on the physics motivation and requirements for observables and measurements will be given for selected examples.

\subsection{Diffractive processes}

The total cross-section for $p p$ interactions can be divided into several classes of events, as shown also schematically in Fig. 2 (below also a rough estimate of the fractional contribution to the total cross-section $\sigma_{\text {tot }}(p p)$ is given):

- elastic scattering $(p p \rightarrow p+p)$, about $30 \%$ of $\sigma_{t o t}(p p)$,

- single diffractive dissociation $(p p \rightarrow p+X)$, about $10 \%$ of $\sigma_{\text {tot }}(p p)$,

- double diffractive dissociation $(p p \rightarrow X+Y)$, about $4 \%$ of $\sigma_{t o t}(p p)$,

- central diffractive dissociation $(p p \rightarrow p+X+p)$, about $1 \%$ of $\sigma_{t o t}(p p)$,

- non-diffractive inelastic scattering $(p p \rightarrow X)$, about $55 \%$ of $\sigma_{t o t}(p p)$.

A characteristic feature of diffraction is the occurence of so called large rapidity gaps, which are regions in phase space without particle production (indicated in the above list of processes by the ${ }^{\prime}+^{\prime}$ sign). Except for double diffractive dissociation, diffractive events also contain one (or two) leading protons (protons which have lost only a small fraction of their momentum).

The momentum loss $\xi$ of a proton in single diffraction $(p p \rightarrow p+X)$ is related to the mass $M_{X}$ of the dissociative system $X$ by the relation

$$
M_{X}^{2}=\xi \cdot s
$$



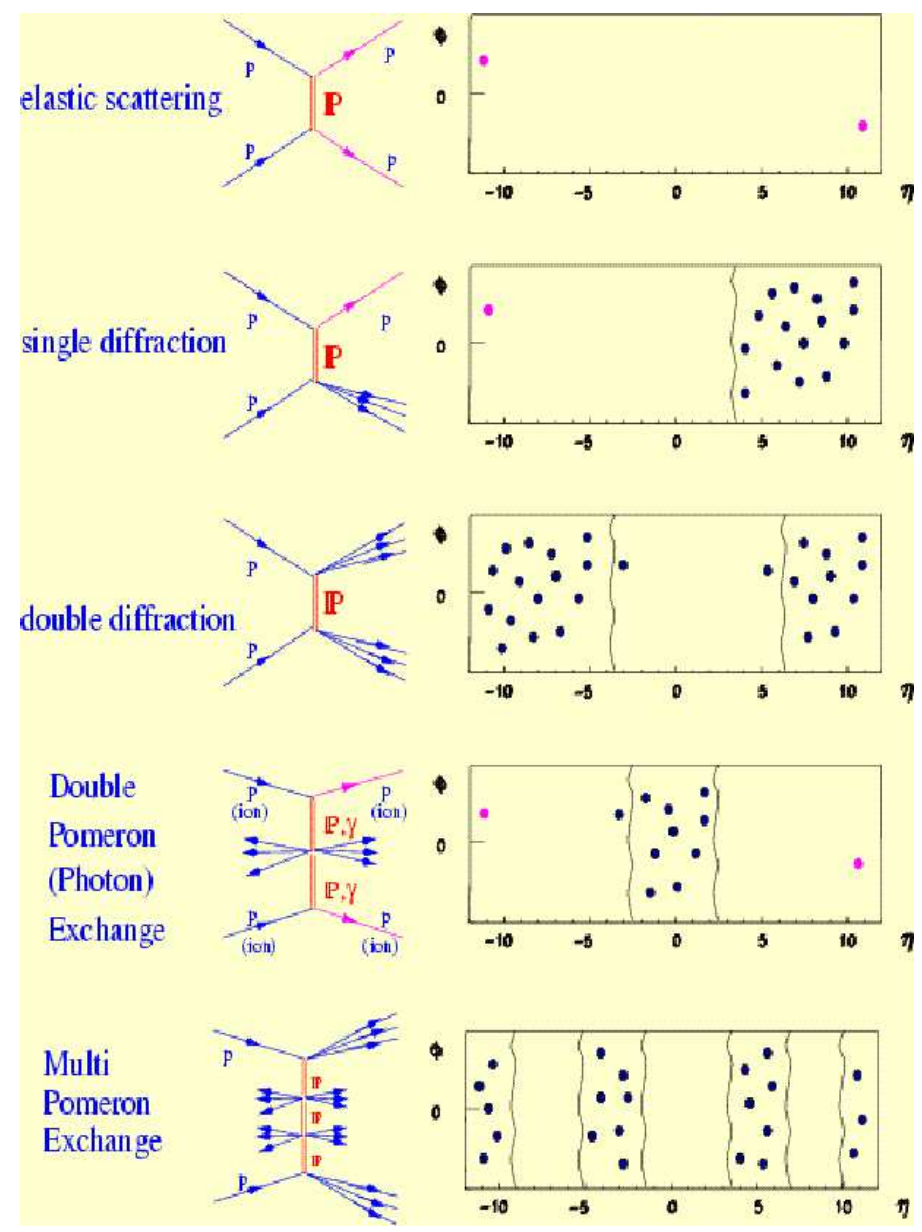

Figure 2: Diffractive processes in proton-proton collisions.

where $s$ denotes the square of the center-of-mass energy. The distance in pseudo-rapidity $\Delta \eta$ between the leading proton and the closest particle belonging to the system $X$ is given by

$$
\Delta \eta \approx-\log \xi
$$

In non-diffractive processes, the occurence of large rapidity gaps is exponentially suppressed with increasing values of $\Delta \eta$, due to the colour flow in the interaction.

Most of the cross-section for diffraction is given by soft processes, where no hard scattering occurs. A precise understanding of the properties of soft diffractive events and of the fractional contribution of the various diffractive event classes to the total cross-section is important for a precise measurement of the total cross-section itself. The knowledge is also of relevance for an improved understanding of cosmic ray physics, as discussed further below.

The description of soft diffractive processes relies mostly on phenomenological models (e.g. by Regge theory), whereas the occurence of a hard process in a diffractive event (e.g. the production of a high $p_{T}$ jet) allows to investigate the partonic structure of these processes, and thus possibly obtain a description from first principles (from the Lagrangian 


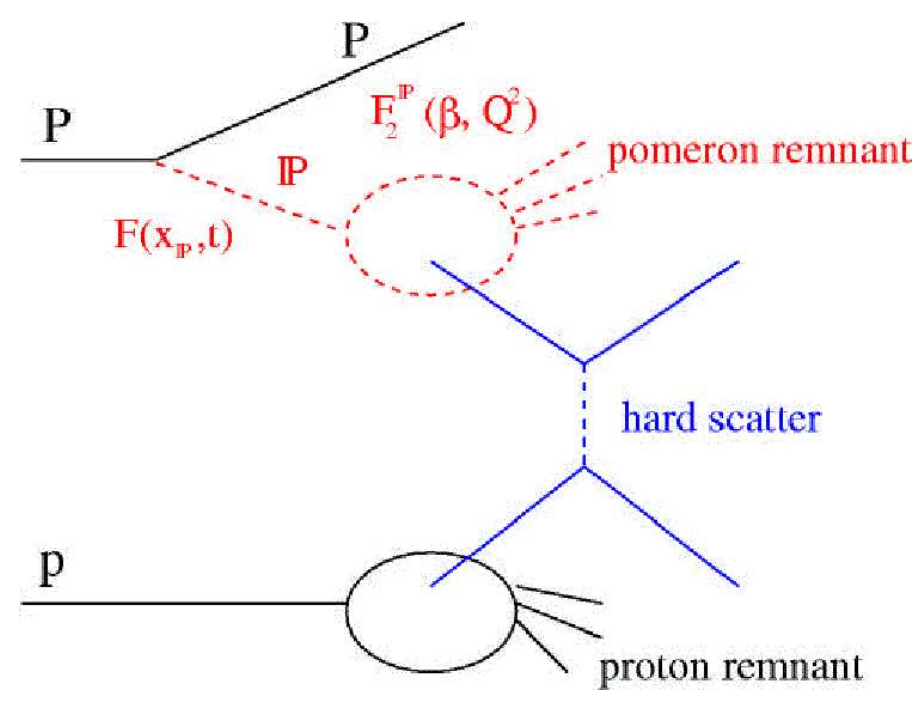

Figure 3: Hard scattering in single diffractive dissociation.

of QCD). Extensive data are available from electron-proton scatterning at HERA [2]. The comparison of these data to hard diffractive events, as measured in $p \bar{p}$ interactions at Tevatron [3], has led to interesting observations. A breakdown of factorisation ${ }^{2}$ is found, which might be related to the survival probability of rapidity gaps. As shown in Fig. 3 , a hard process in single diffractive $p p$ scattering can be visualized as the interaction of two partons, one of which belongs to a proton and the other forms part of a colour-less entity coupling to the other, quasi-elastically scattered proton. This entity is called Pomeron, in reference to the Pomeranchuk trajectory describing the properties of hadron-hadron interactions at high energy within Regge theory. Within the parton model and QCD, the Pomeron could be modelled as a two gluon system, in a colour singlet configuration. The LHC with its increase center-of-mass energy and its high luminosity should provide a wealth of data to obtain better insight in this class of processes within the strong interaction.

\subsection{Exclusive production to search for new physics}
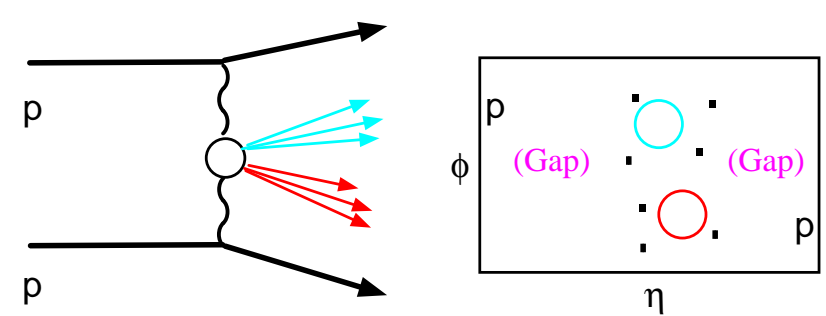

$\eta$

Central diffractive processes, as shown in Fig. 目, lead to the production of a central system (e.g. containing two jets as shown) and two leading protons, each of them separated by a gap in rapidity from the central system. Due to its large center-ofmass energy, the LHC can be thought of providing Pomeron-Pomeron collisions with a broad reach in the Pomeron-Pomeron center-of-mass energy, extending up to $\mathcal{O}(\mathrm{TeV})$.

\footnotetext{
${ }^{2}$ implying that diffractive parton densities, as determined from HERA data, do no predict correctly the observed cross-section for hard diffractive scattering at Tevatron.
} 
If the momentum loss $\xi_{1,2}$ of both protons is measured precisely, then the mass $M_{X}$ of the central system can be determined (via the so called missing mass method [何) as

$$
M_{X}^{2}=\xi_{1} \cdot \xi_{2} \cdot s .
$$

At LHC, as an example, a central system with a mass of $140 \mathrm{GeV}$ requires $\xi_{1} \cdot \xi_{2}=10^{-4}$. This can be realized by having both protons lost $1 \%$ of their momentum (symmetric configuration) or e.g. by having one proton with a momentum loss of $\xi_{1}=2 \cdot 10^{-3}$ and the other one having $\xi_{2}=0.05$. For the symmetric case, the central system is produced at rest in the laboratory frame, otherwise it is boosted along the beam direction.

The process of central diffraction could provide a complementary way to search for and determine properties of a light Higgs boson. As discussed in detail in [0, 6, the exclusive production of a Higgs boson would provide a very clean signature and would allow to use the decay mode $H \rightarrow b \bar{b}$ (having the largest branching ratio for masses around $120 \mathrm{GeV}$ ). Due to selection rules, it is expected that the signal-to-background ratio will very favourable. However the expected cross-section is not too large, about $3 \mathrm{fb}$, although the uncertainties on this value are not negligible. The detection and precise measurement of both protons could allow for a mass resolution of $\mathcal{O}(1 \mathrm{GeV})$. It is also possible that other new particles might be detected and measured in exclusive production, e.g. pairs of super-symmetric particles.

Exclusive production can not only occur via strong interaction processes, but also in two photon processes. As discussed in [7], this process could also be used for exclusive production of the Higgs boson, as well as of $W^{+} W^{-}$pairs or $t \bar{t}$ pairs.

\subsection{Low $x$ physics}

The study of parton dynamics at small values of $x^{3}$ might reveal additional insight into the dynamics of the strong interaction and its description by QCD. This can be done possibly by measurements of the proton structure function (or the various parton densities) or by studying specific final states, such as forward jet production. Fig. 7 indicates the reach of existing data from fixed target experiments and of the HERA experiments in the plane of Bjorken- $x$ and squared momentum transfer $Q^{2}$ (of the hard scattering). The baseline reach of LHC extends for a given value of $x$ to much larger values of $Q^{2}$, thus insuring the validity of the perturbative approach. On the other hand, for fixed values of $Q^{2}$, smaller values of $x$ can be reached than e.g. at HERA. As discussed later, the acceptance of e.g. ATLAS and CMS for high $p_{T}$ objects is restricted to the region of $|\eta|<2.5$ (for leptons, photons and $b$-tagged jets) and up to $|\eta|<5$ (for jets). As can be seen from the diagram, in order to reach values of $x \approx 10^{-6}$ or smaller, one would have to measure in the region $5<|\eta|<8$ (for values of $Q^{2}>10 \mathrm{GeV}^{2}$ ). It is thus clear that the smallest values of $x$ will only be accessible if coverage for large values of the pseudo-rapidity $|\eta|$ is available.

For physics at small $x$ values, it is also important to point out the studies which can be done in $p A$ and $A A$ collisions, which can help to understand better the structure and the dynamics of nuclear matter. Due to the large number of partons in nuclei for small $x$ values, saturation and shadowing effects might be more easy to observe in these collisions.

\footnotetext{
${ }^{3} x$ being the Bjorken- $x$, indicating the momentum fraction of a parton out of the proton.
} 


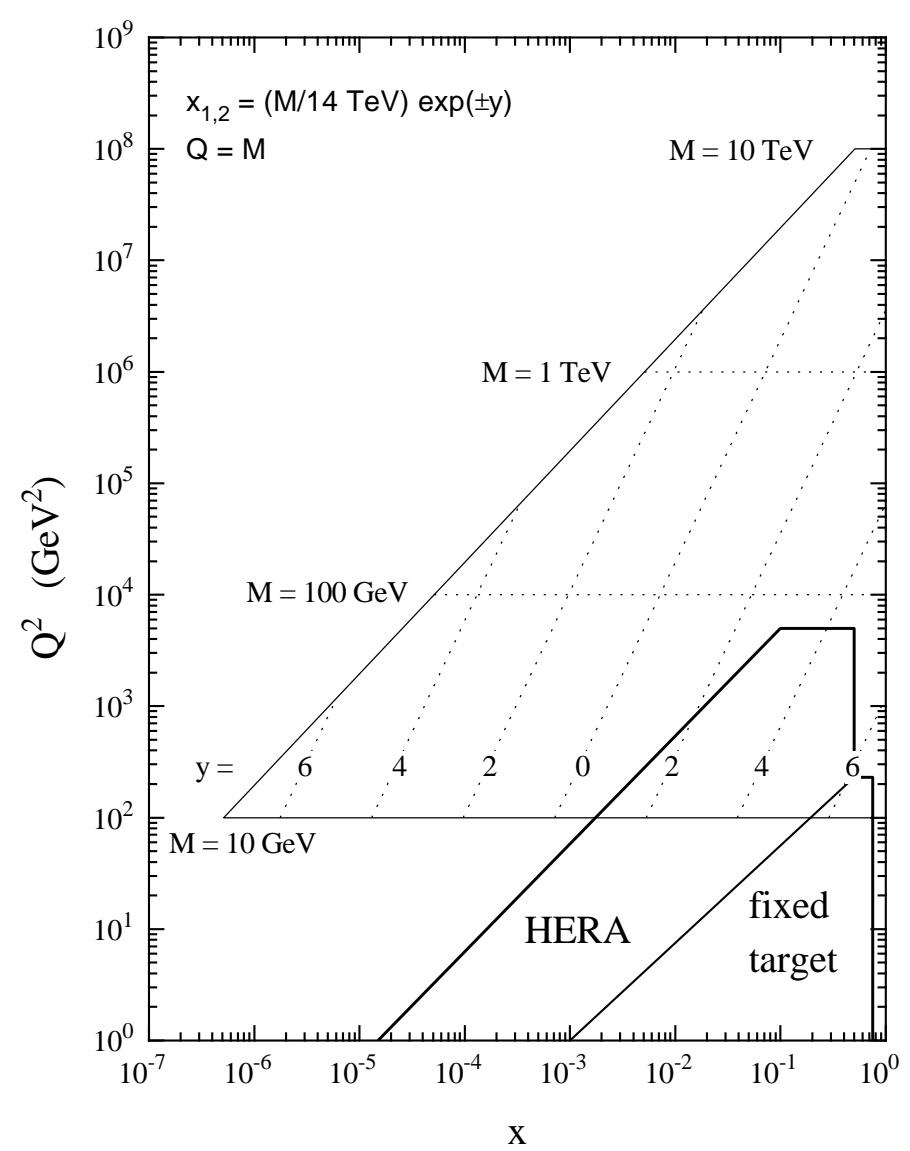

Figure 5: Kinematic reach in the $\left(x, Q^{2}\right)$ plane, indicating the LHC coverage for various acceptances in rapidity $y$.

\subsection{Event structure and cosmic ray physics}

In Fig. 6, the flux of cosmic rays is shown as determined from extended air showers created in the earth atmosphere. The observed spectrum extends in a power-law form over many orders in magnitude, more than 10 in energy and more than 30 in flux, without showing any clear structures. Of very special interest [ [8] are events seen at the upper end of the spectrum, with energies of more than $10^{19} \mathrm{eV}$. The LHC will probe the energy region of about $10^{17} \mathrm{eV}$ in $p p$ collisions and about $10^{18} \mathrm{eV}$ in $\mathrm{PbPb}$ collisions, extending the reach by up to three orders of magnitude beyond the one of Tevatron.

It is important to note that the available statistics at LHC will be enormous, in comparison to the observed rate of cosmic rays in this energy regime. For the region of the so called ankle (about $10^{18} \mathrm{eV}$ ), only one cosmic ray event is expected per $\mathrm{km}^{2}$ and per year, whereas at LHC a rate of $1 \mathrm{~Hz}$ of accepted events will provide a sample of $10^{7}$ events per year. 


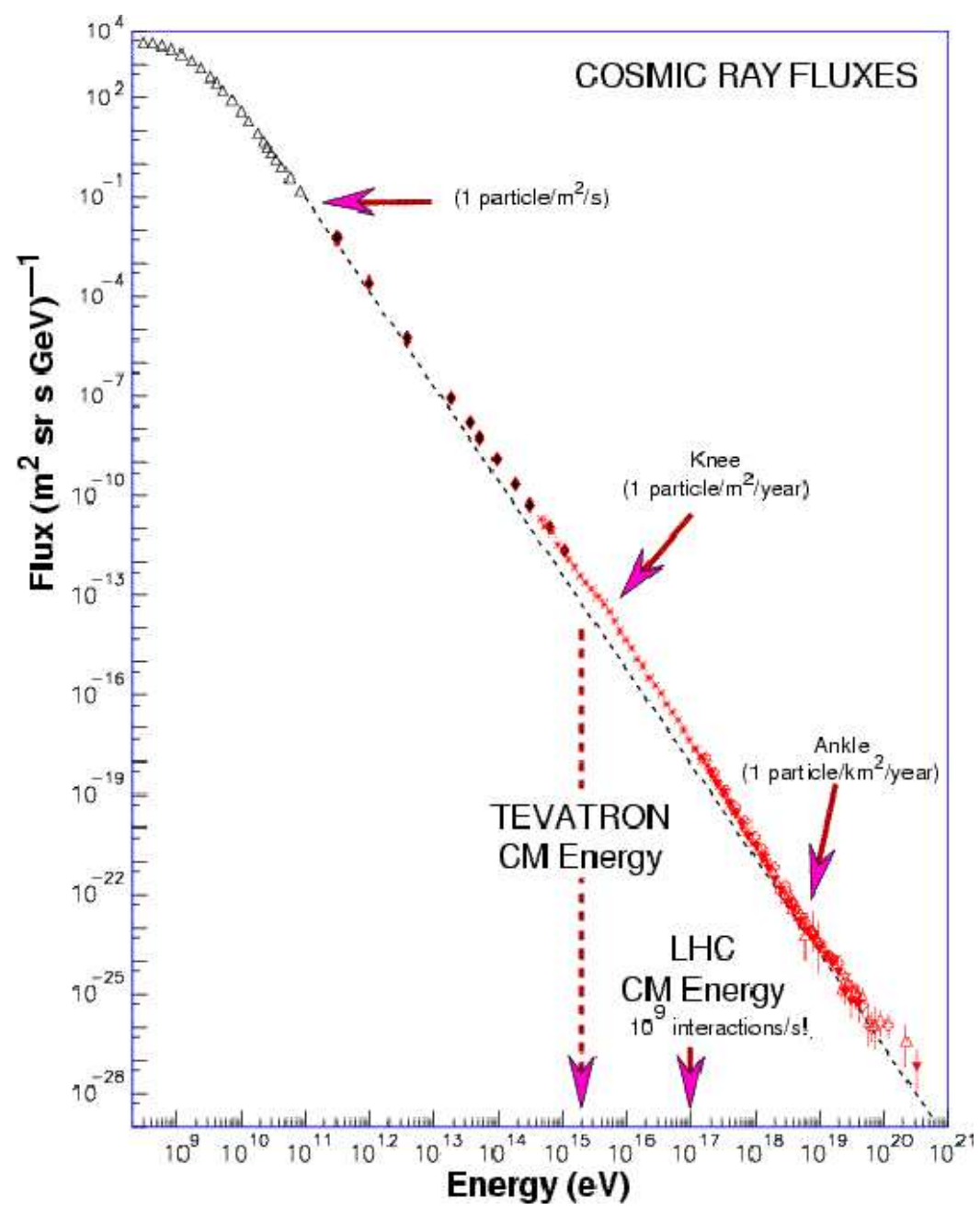

Figure 6: Cosmic ray flux.

The interpretation of the extended air showers observed on the earth's surface aims at a precise determination of the energy and of the species of the incident particle initiating the shower. This unfolding from the observed particles and their properties needs however precise models of the hadronic interaction, which in turn rely on extrapolation from existing accelerator measurements. It is expected that the uncertainties will be reduced once the range of extrapolation is getting smaller.

As an example, Fig. 7 shows the fractional energy $x_{l a b}=E / E_{l a b}$ of leading hadrons produced in $p \bar{p}$ collisions at a proton energy $E_{l a b}=10^{17} \mathrm{eV}$. Clearly visible are the differences in the prediction of the four models shown. The existing data do not constrain enough the hadronic interactions models and further measurements at higher energy are most welcome.

Some of the most important measurements to be performed at the LHC include the total $p p$ cross-section, the fraction of diffractive dissociation in the total cross-section, the energy flow, and multiplicities as well momentum spectra of leading particles.

Of special importance for these measurements is the forward region, as the behaviour 


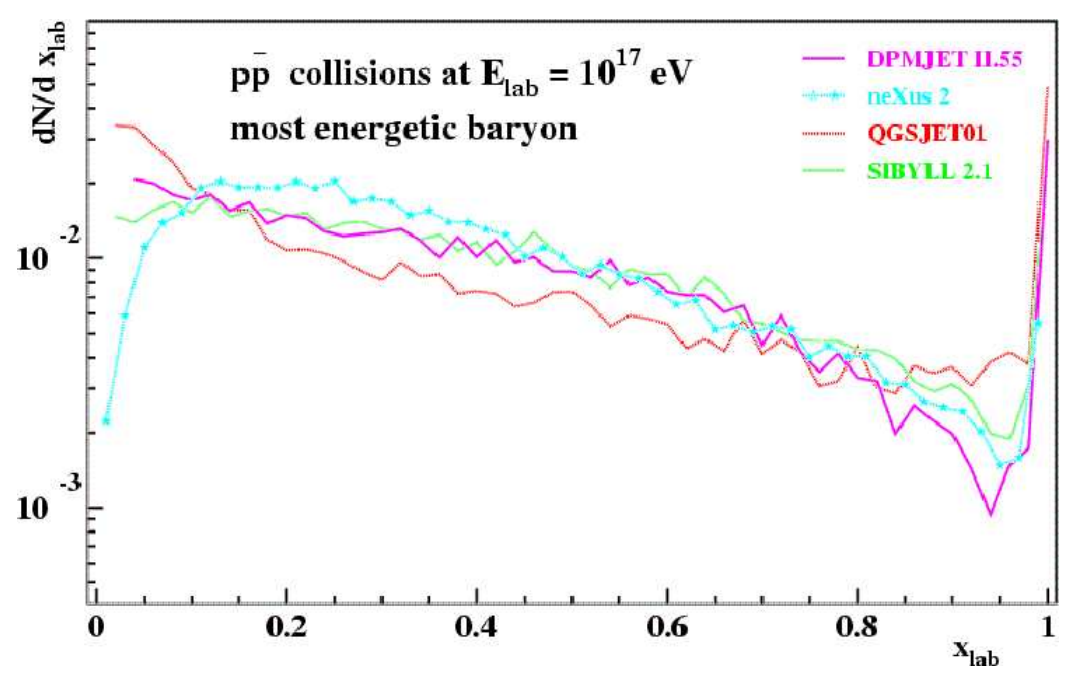

Figure 7: Predictions of various models for properties of $p \bar{p}$ interactions at energies of $E=$ $10^{17} \mathrm{GeV}$, showing the distribution of the fractional energy $x_{l a b}$ for the leading hadron.

of the inelastic interactions and the spectrum of leading particles in this region determines the energy transport through the atmosphere and thus strongly influences the air shower development. It is important to point out that present models indicate that measurements of only the central region (for properties such as energy flow and multiplicities of inelastic events) are not sufficient, as the models do not predict a consistent behaviour between changes in the central region and the forward region. For a more detailed discussion of relevant measurements and their importance, see [9, 10].

\section{LHC machine and running scenarios}

The LHC will be installed in the former LEP tunnel, which is located at up to $100 \mathrm{~m}$ below surface and has a circumference of about $27 \mathrm{~km}$. It will consist of two rings, where the beams can be brought into collision at four interaction points. In order to reach a center-ofmass energy of $\sqrt{s}=14 \mathrm{TeV}$ for $p p$, more than 1200 super-conducting dipole magnets with a nominal field strength of $8.3 \mathrm{~T}$ are needed to bend the protons. The design luminosity will be $\mathcal{L}=10^{34} \mathrm{~cm}^{-2} \mathrm{~s}^{-1}$, to be reached by filling the machine with 2835 bunches, each containing about $10^{11}$ protons. The separation between two bunch crossings will be $25 \mathrm{~ns}$. It is feasible to run the machine at lower values of $\sqrt{s}$, down to about $2 \mathrm{TeV}$, and thus giving the possibility of obtaining overlap with proton-antiproton collisions at Tevatron.

Furthermore, the LHC is designed to provide nucleus-nucleus collisions. In the case of $\mathrm{PbPb}$ collisions, a center-of-mass energy of $1148 \mathrm{TeV}^{4}$ can be reached at a luminosity of $\mathcal{L}=10^{27} \mathrm{~cm}^{-2} \mathrm{~s}^{-1}$. Collisions of lighter ions are possible as well, e.g. of $S n, K r, A r$ and $O$. In addition, the LHC can operated in $p A$ mode, colliding protons on nuclei. In this case the center-of-mass system of the collision will not be at rest in the laboratory frame, but shifted by up to one unit in rapidity. Luminosities foreseen for $p A$ collisions should range from $\mathcal{L}=7.4 \cdot 10^{29} \mathrm{~cm}^{-2} \mathrm{~s}^{-1}$ for $p P b$ up to $\mathcal{L}=1.0 \cdot 10^{31} \mathrm{~cm}^{-2} \mathrm{~s}^{-1}$ for $p O$.

\footnotetext{
${ }^{4}$ corresponding to an energy of $2.75 \mathrm{TeV}$ per nucleon.
} 


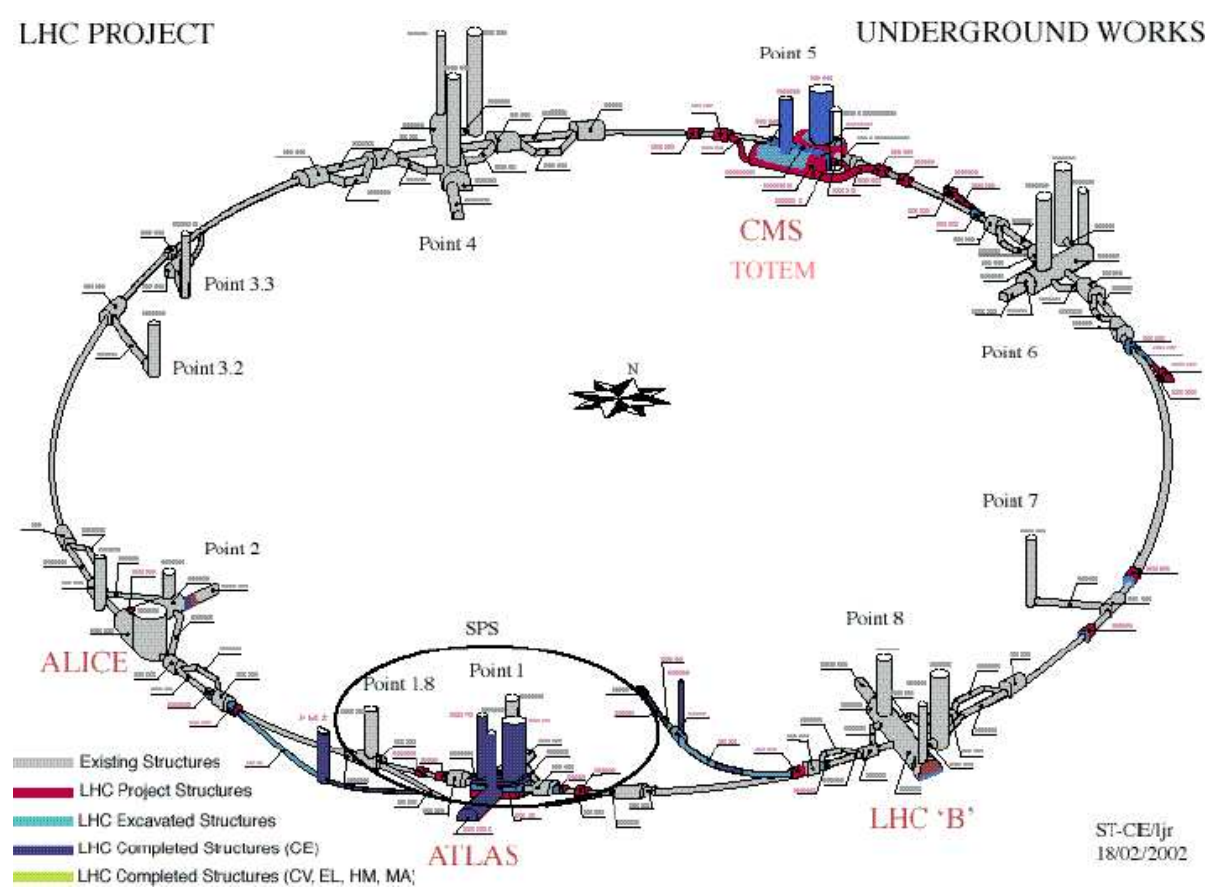

Figure 8: Layout of the LHC, showing the underground caverns of the four interactions regions.

Most of the time, the LHC is expected to be operated in $p p$ mode. Approximately one month per year should be devoted to the studies of nucleus-nucleus and proton-nucleus collisions. Furthermore shorter dedicated runs with special conditions should take place, e.g. for TOTEM to perform a precise measurement of the total cross-section.

\section{LHC experiments}

Two big underground caverns (at the interactions points 1 and 5) have been excavated for the two general purpose experiments, ATLAS and CMS, which are optimized for high $p_{T}$ physics. At interaction point 2, the ALICE experiment will be situated, dedicated to the study of heavy-ion collisions. Point 8 will be taken by LHCb, aiming at the study of $b$-hadron physics. Furthermore, the TOTEM experiment (to be installed in point 5) will measure the total cross-section in $p p$ collisions.

For most experiments, the design phase has been finished and the mass production of their components (esp. in the case of ATLAS and CMS) is well under way, sometimes even close to completion. In the following, a brief overview of the main features of each experiment is given.

\subsection{ALICE}

The ALICE 11] detector, as shown in Fig. 9, will re-use the magnet of the L3 experiment. The central element of ALICE will be a huge time projection chamber (TPC), allowing precise tracking in the high multiplicity environment of central heavy ion collisions. Its coverage will be $|\eta|<1$. Inside the magnet further components are foreseen for photon 


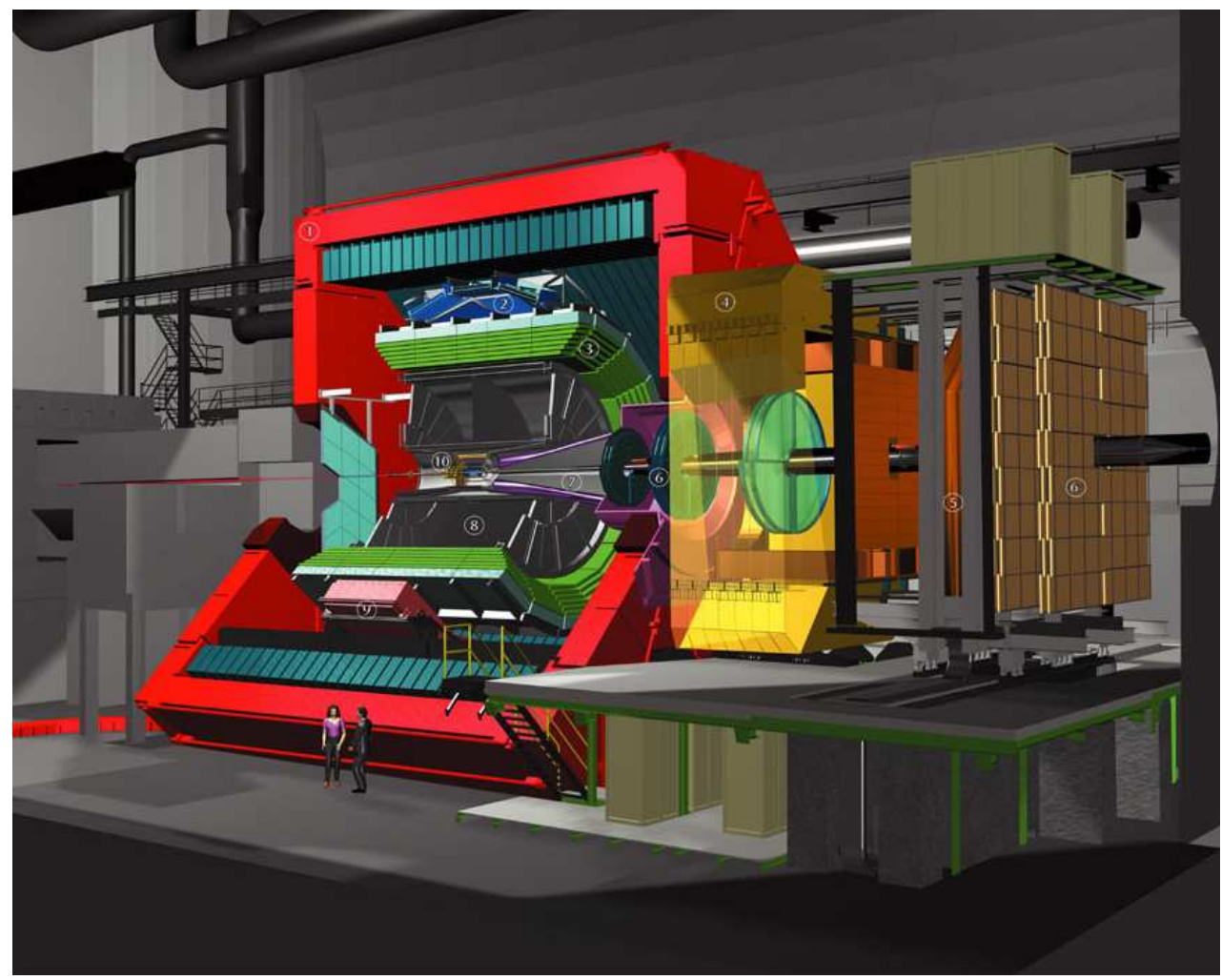

Figure 9: The ALICE detector.

detection, for electron-positron pair detection and for multiplicity measurements (the latter using Si detectors covering the region of $-5.4<\eta<3$ ), as well as for particle identification (via time-of-flight and transition radiation).

Outside of the magnet, a dedicated muon spectrometer $(2.4<\eta<4)$ with a separate dipole magnet is situated on one side of the experiment. ALICE will have also detectors in the machine tunnel: a zero degree calorimeter to measure e.g. the centrality of the heavy ion collision.

\subsection{ATLAS}

ATLAS [12] is a general purpose experiment, shown in Fig. 10, optimized for high $p_{T}$ physics. Surrounding the interaction point, several tracking detectors will measure charged particles and reconstruct (primary and secondary) vertices. Closest to the beam, three layers of $S i$ pixel sensors will be placed, followed by four layers of $S i$ strip detectors. Further out, there will be a straw tube tracker (TRT), which can detect transition radiation to identify electrons. All these components are situated inside a solenoid magnet with a field of $2 \mathrm{~T}$. The tracking detectors (Inner Detector) cover the region up to $|\eta|<2.5$ and are surrounded by calorimetry, extending up to $|\eta|<4$.9. In the barrel region, a fine grained liquid argon ( $\mathrm{LAr}$ ) accordion calorimeter is foreseen as electromagnetic part, followed by a tile scintillator calorimeter as hadronic compartment. In the endcap and forward region, LAr technology is used again. Outside of the calorimeters, an open air-core toroid magnet 


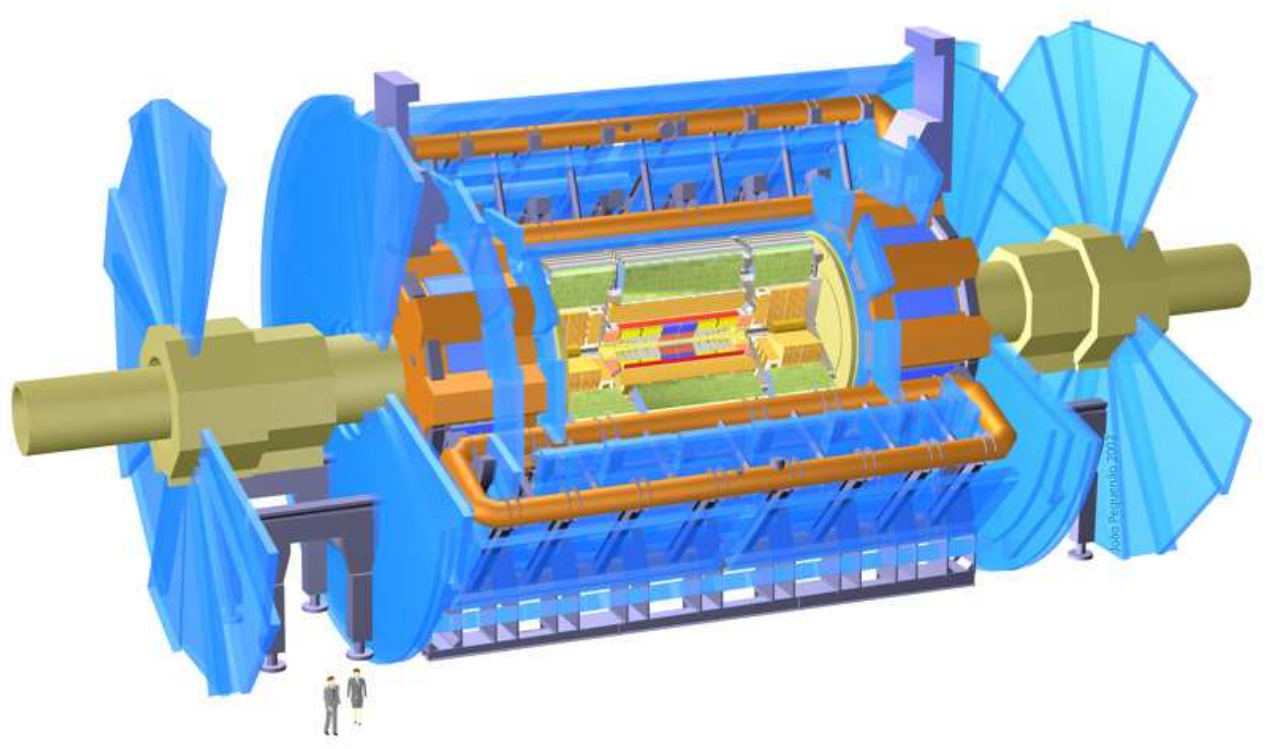

Figure 10: The ATLAS detector.

system is situated, interleaved with muon detectors, to provide detection of muons and a stand-alone measurement of their momentum in the region $|\eta|<2.7$.

The overall size of ATLAS is about $40 \mathrm{~m} \times 22 \mathrm{~m}$ and its weight will be about $7000 \mathrm{t}$. More details on the expected performance of ATLAS can be found in Ref. 13.

\subsection{CMS}

CMS [14] is the other general purpose detector and is shown in Fig. 11. As ATLAS, it has been optimized for the detection of high $p_{T}$ leptons, photons, jets (with and without $b$-tagging) and measurement of missing transverse energy. The tracking is based on an all silicon system, where the interaction point is surrounded with layers of pixel detectors. The remainder of the tracking volume is made of layers of $S i$ strip detectors. The tracking coverage extends up to $|\eta|<2.5$. Surrounding the tracker, a $\mathrm{PbWO}_{4}$ crystal electromagnetic calorimeter is situated, which is followed by a scintillator sandwich hadronic calorimeter. All of these components are located inside a large solenoid magnet, providing a field of $4 \mathrm{~T}$. The calorimetric coverage is extended up to $|\eta|=5$ by a forward calorimeter, instrumented with quartz fibers. The return yoke is instrumented for muon detection, covering the region $|\eta|<2.5$.

CMS will have a size of $22 \mathrm{~m} \times 15 \mathrm{~m}$ and a weight of about $13000 \mathrm{t}$.

\subsection{LHCb}

The LHCb 15 layout (as shown in Fig. 12) resembles a forward spectrometer, although 


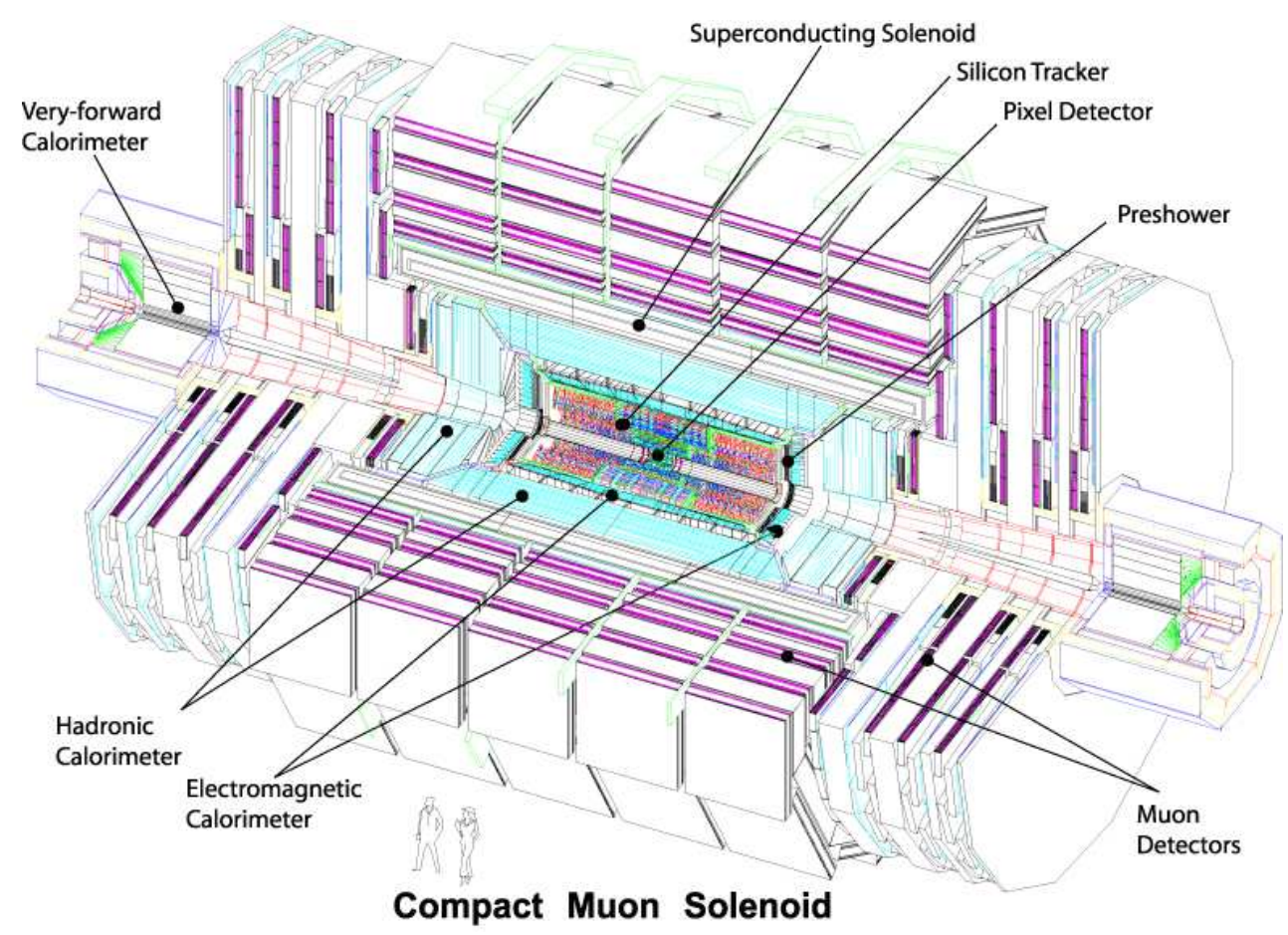

Figure 11: The CMS detector.

LHCb will take data from colliding proton bunches. The interaction point will be surrounded be a precise vertex detector, followed by a tracking system, including a dipole magnet. LHCb will have various possibilities for particle identification, including two ringimaging Cerenkov (RICH) detectors, electromagnetic and hadronic calorimetry and a muon system. The acceptance region extends over $1.9<\eta<4.9$.

\subsection{TOTEM}

The primary goal of TOTEM is to measure the total cross-section via the luminosity independent method, which requires the simultaneous determination of elastic scattering (under small angles) and of the rate for inelastic interactions.

TOTEM will thus have two types of detectors (as shown in Figs. 13 and 14). Firstly, detectors to measure charged particles from inelastic events in the region $3<|\eta|<7$ and secondly, detectors to measure leading protons (e.g. from elastic scattering) at distances of 100 - $200 \mathrm{~m}$ from the interaction point in the machine tunnel (using so called Roman Pots). TOTEM will be installed in interaction point 5 , the inelastic detectors will be located inside the CMS experiment.

\subsection{Baseline coverage for forward physics}

The baseline design of the experiments, as described above, will allow (although not always in the same experiment) to measure the production of identified particles in the region $-2.5<\eta<4.9$, where ATLAS and CMS should be able to reach $p_{T}$ values of $\mathcal{O}(1 \mathrm{GeV})$ for 


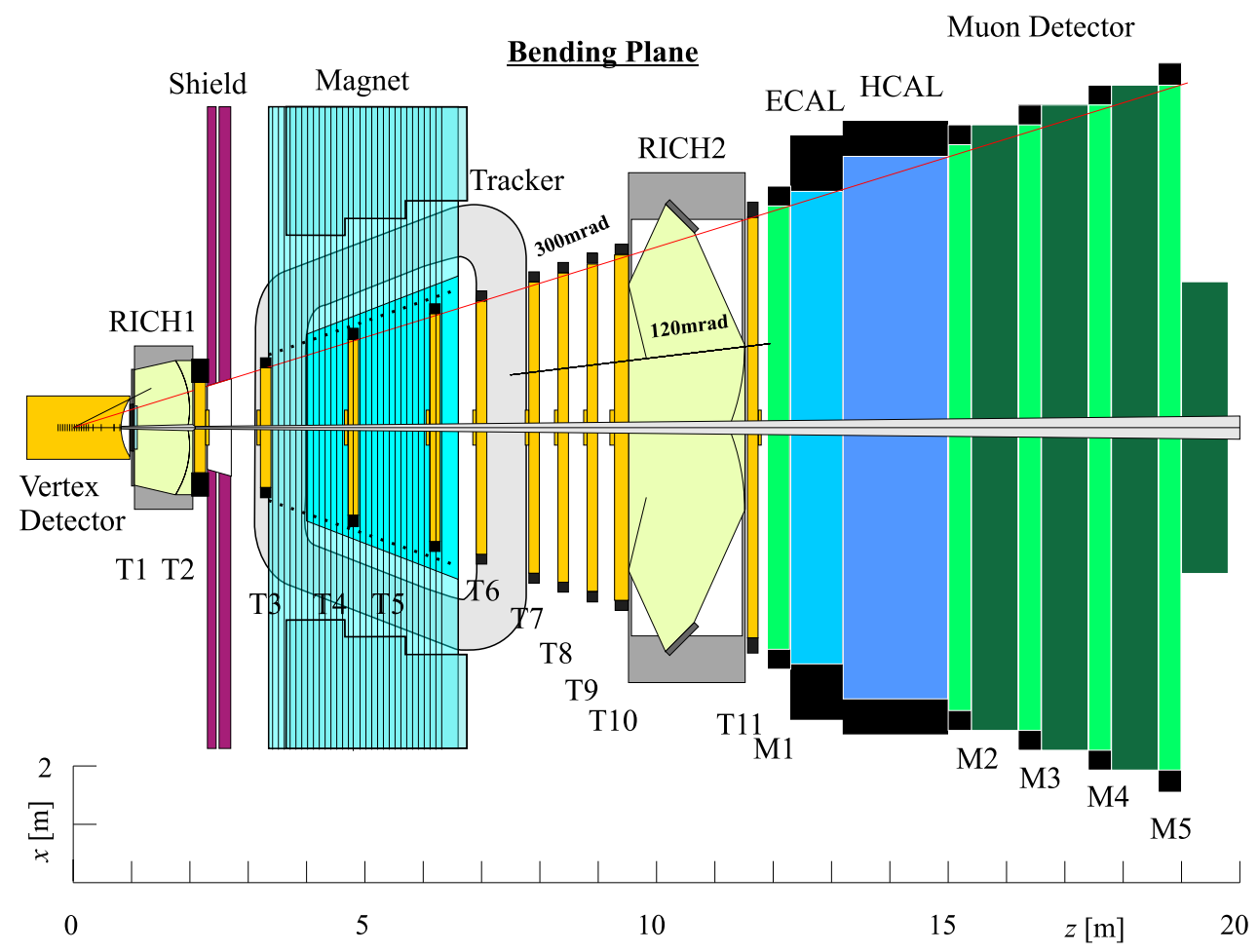

Figure 12: The LHCb detector.

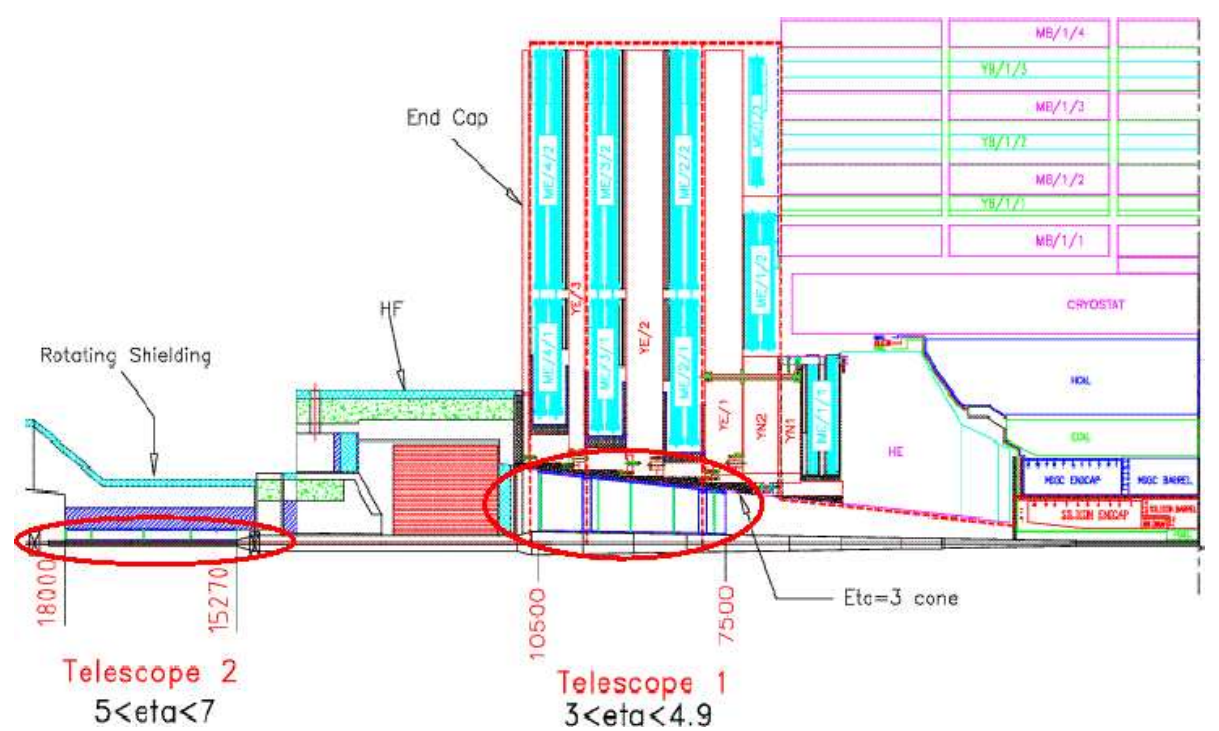

Figure 13: The TOTEM layout of the two telescopes for measurement of particles from inelastic interactions within the CMS detector.

the region of $|\eta|<2.5$. Both ALICE and LHCb will extend this reach down to $\mathcal{O}(0.1 \mathrm{GeV})$, although mostly only in the regions $|\eta|<1$ (ALICE) and $1.9<\eta<4.9$ (LHCb).

Furthermore, the charged multiplicity will be measured by ALICE in the region $-5.4<$ $\eta<3$ and by TOTEM in the region $3<|\eta|<7$. The energy flow will be covered by ATLAS 


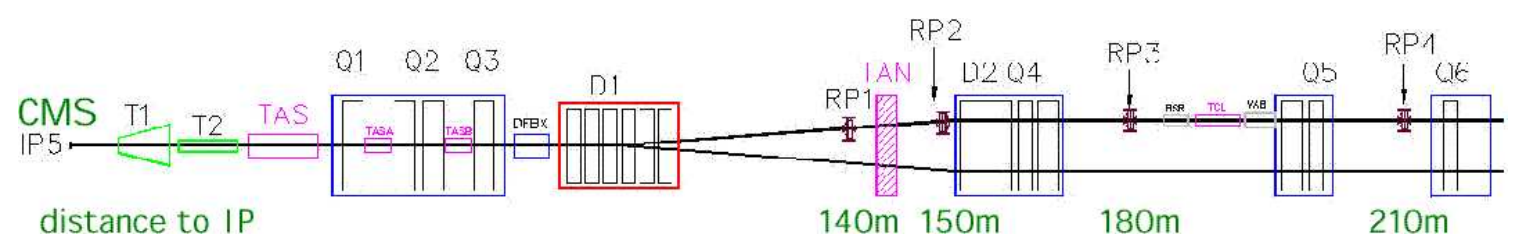

Figure 14: The TOTEM layout for the leading proton measurements, showing four possible locations (RP1 - RP4) for Roman Pots.

and CMS for $|\eta|<5$. ALICE will be able detect leading neutrons and TOTEM will measure (at least during dedicated runs) leading protons as well.

\section{Possibilities for extensions of coverage}

Although two types of possible extensions in the detector coverage can be distinguished, i.e. the measurement of leading particles and the detection and measurement of particles produced under small angles in inelastic interactions, several aspects of instrumentation will be similar, as in both cases the detection has to be done close to the beam (pipe). The measurement of leading particles has to occur at large distances from the interaction point, as these particles are either scattered under very small angles or have lost only a small fraction of their momentum and thus leave the beam envelope only far away from their production point. An increase of the acceptance for particles from inelastic events has to happen mostly within the experimental caverns (before the first magnetic elements of the accelerator) and thus needs to be done very close to the beam, in order to have access to small scattering angles.

\subsection{Extensions within the experimental cavern}

As discussed above, amongst the motivations for extending the coverage, inside the experimental cavern, for the detection of particles from inelastic interactions are the measurements of

- energy flow,

- charged particle multiplicity,

- jet production,

- electron and photon production,

- tagging of rapidity gaps and

- muon multiplicity in $\eta$ regions.

For the measurement of charged multiplicity (where the combination of all experiments should cover up to $|\eta|<7$ ), an extended coverage for the measurement of the energy flow (up to $|\eta|<8$ ) could be achieved by installing additional calorimeters inside the experimental cavern. This would have to be done close to the beam pipe at a distance of about $18 \mathrm{~m}$ 


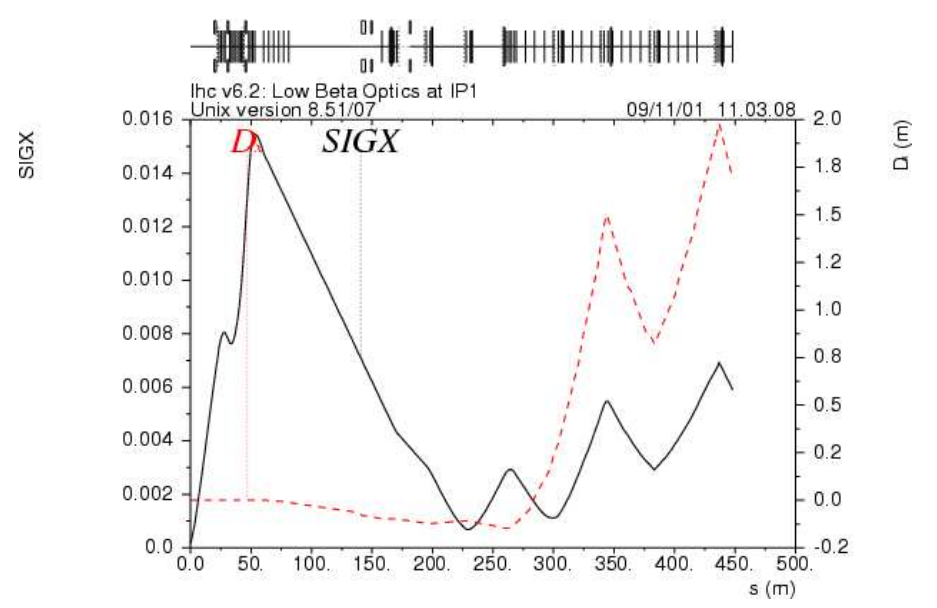

Figure 15: Dispersion $D X$ and $S I G X=10 \cdot \sigma_{x}$ (where $\sigma_{x}$ is the beam size) in the horizontal plane for the high luminosity machine optics set-up.

from the interaction point (or possibly by instrumenting the TAS absorber ${ }^{5}$. A detailed proposal for a very forward calorimeter (surrounding the beam pipe) has been worked out in the context of CASTOR [17], which has been designed primarily to search for centauros and strange objects in heavy ion collisions at LHC. The availability of both a calorimeter and tracking detectors in front of it would allow for limited particle identification capabilities, such as measurements of electrons and possibly also photons.

Instrumentation inside the experimental caverns close to the beam pipe has to take into account the high radiation environment, the limited access possibilities and the need to provide services (e.g. power and signal cables, cooling circuits) to the components. All of this has to respect the already mostly finalized design of the approved experiments.

\subsection{Extensions within the machine tunnel}

The measurement of elastic scattering down to very small values of the momentum transfer $-t$ (which is necessary for a precise determination of the total cross-section, as discussed in [16]) requires a special optics set-up of the machine, where the beams are no longer strongly focused at the interaction point (to obtain the highest luminosity). The layout of the interaction regions 1 and 5 allows for instrumentation to be installed at distances of about $150 \mathrm{~m}$ and $210 \mathrm{~m}$ from the interaction point (see Fig. 14). For this special optics set-up, elastic scattering should be measurable down to values of at least $-t \approx 10^{-2} \mathrm{GeV}^{2}$.

\subsubsection{Acceptance for leading protons}

Protons, which have lost a small fraction of their momentum, are subject to a stronger bending force in the magnetic elements of the machine and thus deviate from the nominal orbit. At large distances from the interaction point, they leave the beam envelope and can thus be detected in sensors operating close to the circulating beam. The displacement $x$ at a given location $s$ along the machine ring depends on the value $\xi$ of the momentum loss

\footnotetext{
${ }^{5}$ situated at the transition between the experimental caverns of ATLAS or CMS and the machine tunnel
} 

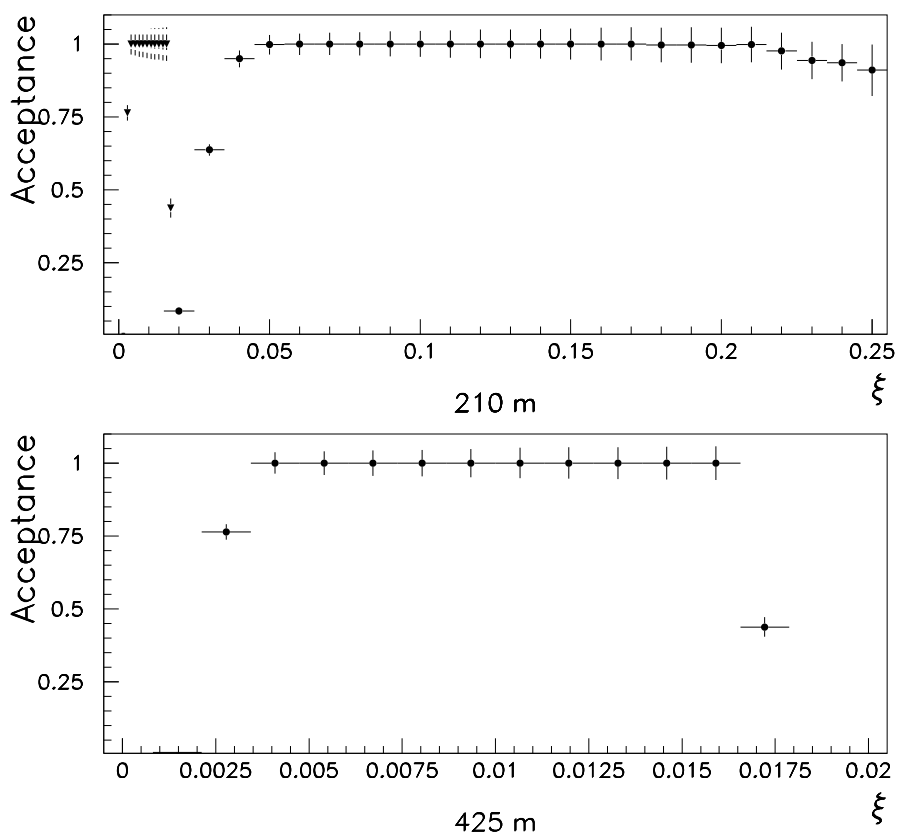

Figure 16: Acceptance for leading protons in the high luminosity machine optics set-up.

and the size of the dispersion $D X$, given by the machine optics, according to

$$
x=D X(s) \cdot \xi \text {. }
$$

In Fig. 15, the evolution of the dispersion (and of the beam size) in the horizontal plane is shown for the nominal LHC optics set-up (strong focusing at the interaction point for high luminosity $-\beta^{*}=0.5 \mathrm{~m}$ ). In order to detect protons with small momentum losses, locations very faw away from the interaction point are required. A proton with a momentum loss of $0.5 \%$ will be displaced by about $5 \mathrm{~mm}$ only at a distance of about $400 \mathrm{~m}$ from the interaction point. Here the beam has a size of about $\sigma_{x}=0.3 \mathrm{~mm}$ in the horizontal plane and thus an approach to a distance of 10 times the beam size would allow to observe such a proton.

In addition, the instrumentation in this region would give coverage for diffractively scattered protons with a momentum loss of about $2 \%$ or more.

A detailed study on the acceptance as a function of the momentum loss $\xi$ of leading protons for two locations (at $215 \mathrm{~m}$ and $425 \mathrm{~m}$ from the interaction point) is shown in Fig. 16. Here an approach to the center of the circulation beam of value of 10 times the size of the beam has been assumed, including an additional distance of $0.1 \mathrm{~mm}$ for inactive areas in the detector. The location presently available for instrumentation at $215 \mathrm{~m}$ allows for values $\xi>0.03$ only (at $50 \%$ acceptance), corresponding to a lower limit of about $420 \mathrm{GeV}$ in mass $M_{X}$ for exclusive central production.

In order to reach smaller values in the momentum loss (and thus in the mass of the centrally produced system), one would have to go to distances of about $425 \mathrm{~m}$, where however presently no warm space for instrumentation is foreseen. At this location the lower limit in $\xi$ is about $2 \cdot 10^{-3}$, corresponding to $M_{X}>28 \mathrm{GeV}$. The upper limit in 


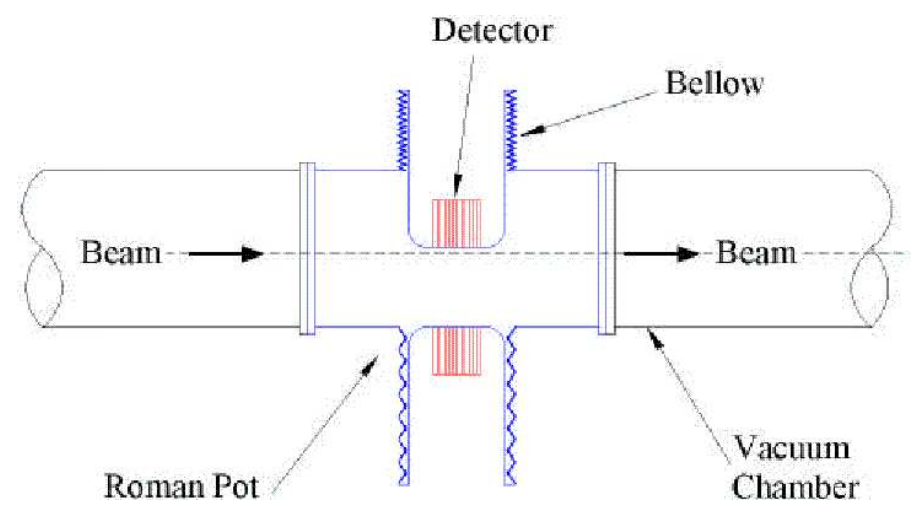

Figure 17: Sketch of a Roman Pot station.

momentum loss is - for a given location - determined by the apertures of the beam pipe and the machine element between the interaction point and the location of the Roman Pot.

\subsubsection{Leading neutrons}

The detection of leading neutrons can be performed in a so called 'zero degree' calorimeter, which would be installed after the beams are separated (to match to the two beam pipe structure in the arcs of LHC). ALICE foresees to have such a device, as mentioned above. For ATLAS and CMS, studies are ongoing which investigate the possibility of instrumenting an absorber (TAN) at a distance of about $140 \mathrm{~m}$ from the interaction point.

\subsection{Instrumentation aspects}

Leading protons have been and are usually measured using silicon or scintillating fibre detectors, located in a movable casing (called Roman Pot), which provides also the separation from the beam vacuum. Fig. 17 shows a schematic drawing of a Roman Pot station, where the beam can be approached from two sides using detectors situated in movable pots. After stable beam conditions are reached, the pots are moved as close as possible towards the circulating beam to provide the best acceptance for small angle elastic scattering as well as for small momentum loss protons.

As the available space for additional instrumentation will often be very limited (e.g. inside the experimental caverns), a new detector concept has been developed, the microstation [18]. Its conceptual design is shown in Fig. 18. The basic idea is to perform the measurement inside the beam pipe, to obtain the closest possible approach of the sensor to the circulating beam. The design aims at a lightweight and very compact component (integrated with the beam pipe). It has to respect several requirements from the machine point of view, such as the compatibility with the machine vacuum and no significant additional impedance to be introduced by the components. The sensor planes will be very precisely movable in a reproducible way, implemented by using inchworm motors built out of ceramic elements. This movement has to be also extremely reliable, as the microstation might be deployed in regions where access is difficult. The sensor is foreseen to be silicon based. Depending on the location and the type of measurement, it could be either of $S i$ strip type 

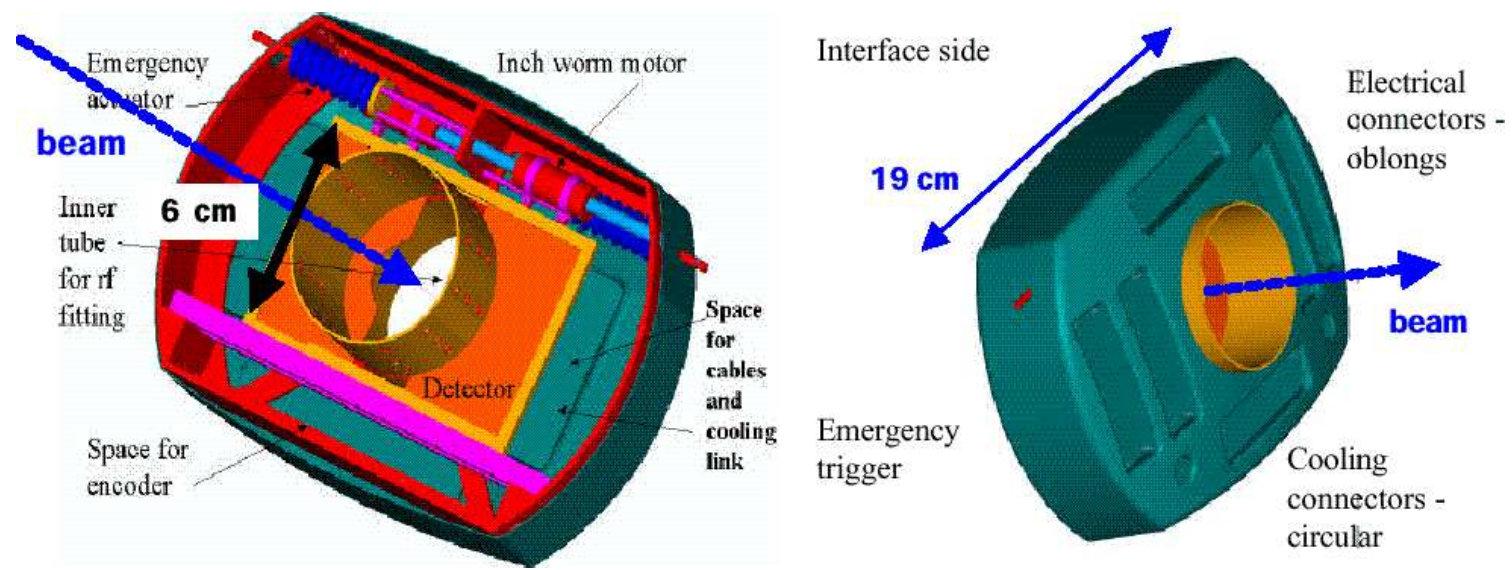

Figure 18: Conceptual design of a microstation.

or of $S i$ pixel type, the latter in case of larger particle densities (i.e. for measurements of inelastic event properties). For these sensors, special emphasis has to be given to a minimisation of inactive areas close to the mechanical edge of the sensor, which would increase the effective distance to the beam center, from where onwards measurements would be possible. A fully functional prototype for validation of the above requirements is presently under construction.

Finally an important issue, which should not be forgotten, is the online selection of events with leading protons. For ATLAS and CMS, the trigger system has to reduce the bunch crossing frequency of $40 \mathrm{MHz}$ (or the interaction rate of about $1 \mathrm{GHz}$ at design luminosity) to a rate of $\mathcal{O}(100 \mathrm{~Hz})$ to mass storage. The first stage of the selection will be a hardware based trigger, which has to operate within a maximum latency of $2.5 \mu \mathrm{s}$ (ATLAS) resp. $3 \mu \mathrm{s}$ (CMS). Events which are not accepted at this first level trigger are lost. Calculating the time-of-flight for protons from the interaction point to the detector position and the signal propagation time back to the electronics cavern of the experiment (where the final trigger decision is made) shows that only leading proton detectors at distances of up to $200-230 \mathrm{~m}$ will be able to deliver in time an input signal to the first level trigger. For locations at large distances, one would possibly have to make a selection based on topological criteria of centrally produced high $p_{T}$ objects and then include the information from leading proton detectors at the higher level trigger stages, where the latency is much less of a constraint.

\section{Conclusions}

The LHC will be in a few years the energy frontier in accelerator particle physics and will offer unique opportunities for studies of the strong interaction in as yet uncovered kinematical regions. In order to maximally exploit the physics potential, extensions of the approved detectors in the forward region (to detect and measure particles scattered under small angles wrt the beam direction) are highly desirable. Such extensions can be classified either as components dedicated to the measurement of leading particles (which then have 
to be installed at large distances of $\mathcal{O}(100 \mathrm{~m})$ from the interaction point) or as detectors for the identification and measurement of particles from inelastic events, produce under small scattering angles (to be installed inside the experimental caverns).

These extensions would provide a significant increase in the physics potential of LHC and its experiments. They are however extremely challenging to develop, as a lot of constraints have to be respected (e.g. compatibility with the operation of the machine and the experiments, restrictions on the available space). Several experiments are presently investigating the technical feasibility of such extensions, where also a coherent effort between the machine and the experiments is needed. The experimental groups are open for suggestions and ideas, as well as to contributions from interested groups not yet participating in the LHC.

\section{Acknowledgments}

It is a pleasure for me to thank the organisers for invitation to speak at this workshop and for creating a very pleasant and stimulating atmosphere during the workshop.

\section{References}

[1] FELIX Letter of Intent, CERN/LHCC 97-45 (1997), http://felix.web.cern.ch/FELIX/.

[2] C. Kiesling, these proceedings.

[3] W. Guryn, these proceedings.

[4] M. G. Albrow and A. Rostovtsev, hep-ph/0009336.

[5] A. de Roeck et al., Eur. Phys. J. C 25 (2002) 391.

[6] V. Khoze, A.D. Martin and M.G. Ryskin, Eur. Phys. J. C 26 (2002) 229.

[7] K. Piotrzkowski, Phys. Rev. D 63 (2001) 071502.

[8] H. Rebel, these proceedings.

[9] NEEDS workshop, Karlsruhe, April 2002, http://www-ik.fzk.de/ needs.

[10] R. Engel, Extensive air showers and accelerator data: the NEEDS workshop, hep-ph/0212340.

[11] ALICE Technical Proposal, CERN/LHCC 95-71 (1995), http://alice.web.cern.ch/Alice/.

[12] ATLAS Technical Proposal, CERN/LHCC/94-43 (1994), http://atlas.web. cern.ch/Atlas/Welcome.html.

[13] ATLAS Detector and Physics Performance Technical Design Report, CERN/LHCC 99-14 and 99-15 (1999).

[14] CMS Technical Proposal, CERN/LHCC 94-38 (1994), http://cmsdoc.cern.ch/cms/outreach/html/index.shtml. 
[15] LHCb Technical Proposal, CERN/LHCC 98-4 (1998), http://lhcb.web.cern.ch/lhcb/.

[16] TOTEM Technical Proposal, CERN/LHCC 99-7 (1999), http://totem.web.cern.ch/Totem/.

[17] A.L.S. Angelis et al., Nuovo Cim. 24C (2001) 755.

[18] V. Nomokonov, The microstation concept for forward physics, published in 'Forward physics and luminosity measurements at LHC', K. Huitu, R. Orava, S. Tapprogge and V. Khoze (editors), World Scientific (2001). 